\title{
SEXUALIDADE NA TERCEIRA IDADE: medidas de prevenção para doenças sexualmente transmissíveis e AIDS
}

\author{
M anoela Busato M ottin M ASCHIO ${ }^{a}$, A na Paula BALBIN O ${ }^{b}$, \\ Paula Fernanda Ribeiro DE SOU ZA' ${ }^{\text {c }}$ L uciana Puchalski KALI N KE ${ }^{d}$
}

\section{RESUM 0}

0 objetivo deste estudo foi identificar as medidas de prevenção que os idosos estão utilizando para à prevenção das D oenças Sexualmente T ransmissíveis e Síndrome da I munodeficiência A dquirida. A pesquisa foi de caráter prospectivo, quantitativo e descritivo com uma amostragem intencional, real izada com 98 idosos. Foi aplicado um questionário com perguntas abertas e fechadas, relacionadas à vida sexual dos idosos frequentadores de uma instituição que desenvolve prog ramas para a mel horia da qualidade de vida dos idosos no município de Curitiba, Paraná. D os entrevistados 43\%, relatam fazer uso de al guma medida de prevenção. A realização de prog ramas de prevenção voltados para o atendimento de pessoas com 50 anos ou mais, deve estar atenta às questões de sexual idade no envel hecimento. Os idosos devem ser vistos como indivíduos que possuem desejos, necessidades sexuais e que fazem projetos para o futuro.

D escritores: Sexualidade. Assistência a idosos. E ducação em enfermagem. D oenças sexualmente transmissíveis. Prevenção primária.

\section{RESUMEN}

E I objetivo de este estudio es identificar medidas que los ancianos están utilizando para la prevención de E nfermedades de T ransmisión Sexual y del Síndrome de I nmunodeficiencia Adquirida. La encuesta fue de carácter prospectivo y cuantitativo con una muestra intencional, realizada con 98 personas de edad avanzada. Se aplicó un cuestionario con preguntas abiertas y cerradas, relacionadas con la vida sexual de los visitantes mayores de una institución que desarrolla programas para la mejora de la calidad de vida de las personas mayores en la ciudad de Curitiba, Paraná, Brasil. El 43\% de los encuestados informó que usa alguna medida de prevención. $L$ a ejecución de los programas de prevención se centró en la atención de personas mayores de 50 años o más, deben estar atentos a las cuestiones de la sexualidad en el envejecimiento. L os ancianos deben ser vistos como individuos que tienen necesidades sexuales y deseos, que hacen proyectos para el futuro.

Descriptores: Sexualidad. Asistencia a los ancianos. E ducación en enfermería. E nfermedades de transmisión sexual. Prevención primaria.

Título: Sexualidad en la tercera edad: medidas de prevención de enfermedades de transmisión sexual y SIDA.

\section{ABST RACT}

This study aimed to identify the methods the elderly are using for the prevention of Sexually T ransmitted D iseases (ST D S) and A cquired Immune D eficiency Syndrome (AID S). T his is a prospective, quantitative and descriptive research with an intentional sample, undertaken with 98 elders. A questionnaire was applied with open and closed questions, related to the sexual life of elders who attend an institution that develops programs to the improvement of elderly w ell- being in Curitiba, state of Paraná, Brazil. $43 \%$ of the elders stated that they make use of some prevention method. P revention programs focused on 50 year old or older people must pay attention to the questions of sexuality and aging. E Iders must be considered as individuals who have desires and sexual needs, and who make plans for the future.

D escriptors: Sexuality. OId age assistance. E ducation, nursing. Sexually transmitted diseases. P rimary prevention.

Title: Sexuality in the elderly: prevention methods for ST D s and AIDS.

\footnotetext{
a Enfermeira do Instituto Pasquini de Hemoterapia e H ematologia, Curitiba, Paraná, Brasil.

b Enfermeira da U nidade de Internação do H ospital U niversitário Cajuru, Curitiba, Paraná, Brasil.

c Enfermeira do Hospital M ater D ei, Curitiba, Paraná, Brasil.

d D outora em Ciências da Saúde, Professora A djunta da U niversidade F ederal do Paraná, Curitiba, Paraná, Brasil.
} 


\section{INT RODUÇÃO}

A população brasileira vem envelhecendo, como pode ser observado pela transição demográfica e pela queda acentuada das taxas de mortalidade e de fecundidade ${ }^{(1)}$. A soma desses dois fatores resulta no envelhecimento global, com as pessoas vivendo mais ao mesmo tempo em que menos nascimentos ocorrem. Para a Organização M undial de Saúde (OM S), terceira idade em países em desenvolvimento é constituído por indivíduos a partir dos 60 anos, e em países desenvolvidos, a partir de 65 anos ${ }^{(2)}$.

A ênfase dada sobre 0 envelhecimento da população mundial não é assunto novo. Países desenvolvidos, como Japão, Estados U nidos, Canadá e alguns países da Europa, já convivem há muito tempo com um grande conting ente de idosos. No entanto, em países em desenvolvimento, como Brasil e M éxico, o número de idosos vêm aumentando rapidamente(3).

A população geral de brasileiros, em 1991, era de 169.799 .710 pessoas. D estes 6,3\%, ou seja, 10.722.705 pessoas tinham 60 anos ou mais de idade. Já no ano de 2000, este total aumentou para 14.536.29 pessoas, aumentando a porcentagem para $8,6 \%$, o que significa um aumento de $35,6 \%$ da população idosa no Brasil(4). E stima-se que o Brasil será o sexto país do mundo em número de idosos até 2025 , com $15 \%$ da população brasileira, ou seja, aproximadamente 30 milhões de pessoas dentro desta faixa etária(5).

Com o aumento da população idosa cresce também o número de casos de Síndrome da I munodeficiência Adquirida (AIDS) entre esta popuIação. A problemática do envelhecimento e da AIDS no Brasil passa por uma questão cultural e de exclusão e concentra-se principalmente no preconceito social relacionado ao sexo nesta ida$\mathrm{de}^{(6)}$.

I dentificada em 1981, nos Estados U nidos, a AIDS tornou-se um marco na história da humanidade. No Brasil, de 1980 até junho de 2009 foram diagnosticados 13.665 casos de AIDS em pessoas com 60 anos ou mais. Destes, 8.959 em homens e 4.696 em mulheres ${ }^{(7)}$. N esse sentido, é preciso desmistificar a concepção arraigada na sociedade de que sexo é prerrogativa da juventude e por isso, só o jovem contrai o Vírus da Imunodeficiência H umana (HIV). Pensar que a terceira idade não tem vida sexual ativa é preconceito(8).
Grande parte da sociedade tenta negar a sexualidade do idoso. As pessoas acham "feio", negam-se a aceitar que o idoso possa querer namorar, esquecem que a sexualidade não é só genitalidade e que existe também uma afetividade que é essencial ao ser humano(9). Portanto, não reconhecer os idosos como população de risco, é um fator contribuinte para o aumento do número de casos de HIV entre as pessoas com 60 anos ou mais.

No Brasil, observa-se a crescente porcentagem, de 7\% em 1996 para 13\% em 2004, de idosos infectados por Doença Sexualmente Transmissíveis (DST s), principal mente a A ID $S^{(10)}$. Este aumento se deve a falta de campanhas de prevenção para estes cidadãos, pois os idosos são tidos como assexuados, e a sexualidade, nesta faixa etária ainda é cercada de tabus e preconceitos por parte da sociedade e também dos profissionais de saúde. A prevenção às DST s e AID S nessa faixa etária se torna um desafio para os responsáveis pelas políticas públicas.

0 preconceito e a dificuldade para se estabelecerem medidas preventivas, especialmente no que se refere ao uso de preservativos, ainda são mais graves do que nos outros segmentos populacionais. Provavelmente por esta razão, são elaboradas poucas campanhas para esse público(11).

A possibilidade de um idoso ser infectado pelo HIV parece invisível aos ol hos da sociedade, e também dos próprios idosos, que não tem a cultura do uso do preservativo. T ambém as mulheres, nesta faixa etária, por em geral não poderem engravidar, têm a falsa impressão da inutilidade do preservativo. Há uma falta de identificação do idoso com as campanhas de prevenção da AIDS, que tem sempre como foco 0 jovem. Então, 0 idoso não se considera como um doente em potencial(11).

Ressalta-se que principalmente após o desenvolvimento de drogas que melhoram o desempenho sexual, o uso de prótese para disfunção erétil para os homens e reposição hormonal para as muIheres, os idosos, tornaram-se cada vez mais ativos sexual mente ${ }^{(12)}$. Todo este avanço veio na tentativa de promover qualidade de vida e uma vida sexual ativa na terceira idade. No entanto, a prevenção das DST para os idosos não acompanhou 0 ritmo desta evolução.

Por estas razões, esses indivíduos se expõem, cada vez mais, a situações de vulnerabilidade, que além de estarem relacionadas às atitudes pessoais, se estendem à dificuldade em diagnosticar preco- 
cemente 0 vírus HIV nesta faixa etária. I sto porque nem sempre sua vida sexual é questionada nas consultas, predominando o mito de que têm ritmo sexual diminuído ou já não fazem sexo ${ }^{(13)}$.

Vários estudos enfatizam o conhecimento sobre HIV/ AIDS em jovens, porém há uma falta de infor mação relacionada a AIDS em idosos. Sendo assim, políticas de prevenção para o idoso devem ser constantes, com programas de educação voltados à vivência saudável e plena da sexualidade na terceira idade, fortalecendo as concepções a respeito das DST, e formas de prevenção. D entro deste contexto, o presente estudo teve como objetivo, identificar as medidas de prevenção que os idosos estão utilizando para a prevenção das DST e AIDS.

\section{MÉTODOS}

A pesquisa foi de caráter prospectivo, quantitativo e descritivo com uma amostragem intencional. E ste método de pesquisa tem como propósito buscar frequência, característica, relação e associação entre variáveis, registrar experiências, observações, eventos não usuais, programas e tratamentos. É o início da busca por explicações para descrever, explorar, classificar e interpretar aspectos de fatos ou fenômenos ${ }^{(14)}$.

0 critério de inclusão para a pesquisa foram idosos, de ambos os gêneros, com idade igual ou superior a 60 anos.

Os participantes da pesquisa foram 98 idosos que fazem parte de uma instituição que desenvolve programas para melhoria da qualidade de vida dos idosos, como práticas físicas, sociais e demais atividades de lazer no município de Curitiba, Paraná, Brasil.

Os idosos foram convidados a participar do estudo e, após aceite, assinaram o T er mo de Consentimento Livre e Esclarecido (T CLE), responderam a um questionário com perguntas abertas e fechadas, relacionadas à sua saúde sexual. Os dados obtidos foram tabulados, analisados através de frequência simples e discutidos com a literatura relacionada ao tema.

A tendendo à Resolução 196/ 96 do Conselho $N$ acional de Saúde ${ }^{(15)}$, os participantes foram convidados a participar do trabalho e, após, assinaram o Termo de Consentimento Livre e Esclarecido, garantindo-Ihes 0 anonimato e a confidencialidade dos dados. 0 projeto foi analisado e aprovado pelo $\mathrm{Co}$ mitê de Ética em Pesquisa em Seres Humanos do
Hospital de Clínicas da U niversidade Federal do Paraná com o número 0260.0.208.000-10.

\section{RESULTADOS}

Dos aproximadamente 200 idosos abordados na pesquisa somente 98 aceitaram participar, o que demonstra o quanto ainda é difícil abordar 0 assunto com este público.

0 perfil epidemiológico da população estudada é: a média de idade dos participantes ficou em 68,9 anos, variando entre 60 e 87 anos. A população feminina foi mais receptiva e prevalente na pesquisa, sendo que $64,2 \%$ (63) são do gênero feminino e 35,7\% (35) do gênero masculino. 0 grau de escolaridade nos mostra que $39,7 \%$ (39) dos entrevistados têm ensino médio, 35,7\% (35) têm ensino fundamental, 18,3\% (18) possuem ensino superior, $4 \%$ (4) não respondeu e somente 2\% (2) nunca estudaram.

Quanto ao estado civil, 72,4\% (71) dos entrevistados são viúvos ou divorciados. D estes 54\% (52) de viúvos. U ma quantidade expressiva 58,1\% (57) não tem companheiro e 39,7\% (39) dos entrevistados disseram ter um companheiro atualmente, $2 \%$ (2) não responderam a esta pergunta. 51\% (50) da população estudada disseram ter uma relação sexual nos últimos 12 meses, 47,9\% (47) disseram não ter relação e 1\% (1) não respondeu.

Quando perguntado se consideram necessário utilizar alguma medida de prevenção, observouse que $87,7 \%$ (86) dos entrevistados responderam sim, 8,1\% (8) responderam não e 4\% (4) não quiseram responder a pergunta. Os idosos que utilizam medidas de prevenção foram questionados o porquê achavam importante utilizar estas medidas. Os comentários mais frequentes foram: para se prevenir, porque a AIDS não tem cura, porque a doença está no sangue e não na cara e, principalmente, para não contagiar os outros. D entre os que responderam que não acham necessário o uso de medidas, os comentários mais frequentes foram: confiança no parceiro, porque tem saúde e porque fez exames de sangue.

Quando solicitados para indicarem as medidas de prevenção que conheciam $70 \%$ (69) indicaram a camisinha e $4 \%$ (4) citaram outras formas como: higiene, cuidado com beijo e saliva e não compartilhar seringas. Entretanto, 10,2\% (10) não sabiam indicar nenhuma medida de prevenção e $15,3 \%$ (15) não responderam. 
Com relação ao uso da medida de prevenção, 42,8\% (42) da população estudada afirmaram fazer uso de al gum tipo de medida de prevenção e, igualmente $42,8 \%$ (42) disseram não fazer uso de medida de prevenção 14,2\% (14) não responderam à pergunta. Dentre os que usam, $64,2 \%$ (27) disseram fazer uso da camisinha, $28,5 \%$ (12) não souberam responder e $7,1 \%$ (3) fazem o uso de medicação como medida de prevenção.
Em relação ao acompanhamento médico para cuidar da saúde sexual $72,4 \%$ (71) dizem realizar acompanhamento. Quanto à periodicidade deste acompanhamento observou-se que $32,6 \%$ (32) fazem a cada seis meses (Gráfico 1 ). A o serem questionados se a AIDS tem cura, 65,3\% (64) da população estudada acham que não, 20,4\% (20) acham que a AID S tem cura e 14,2\% (14) não souberam responder à pergunta.

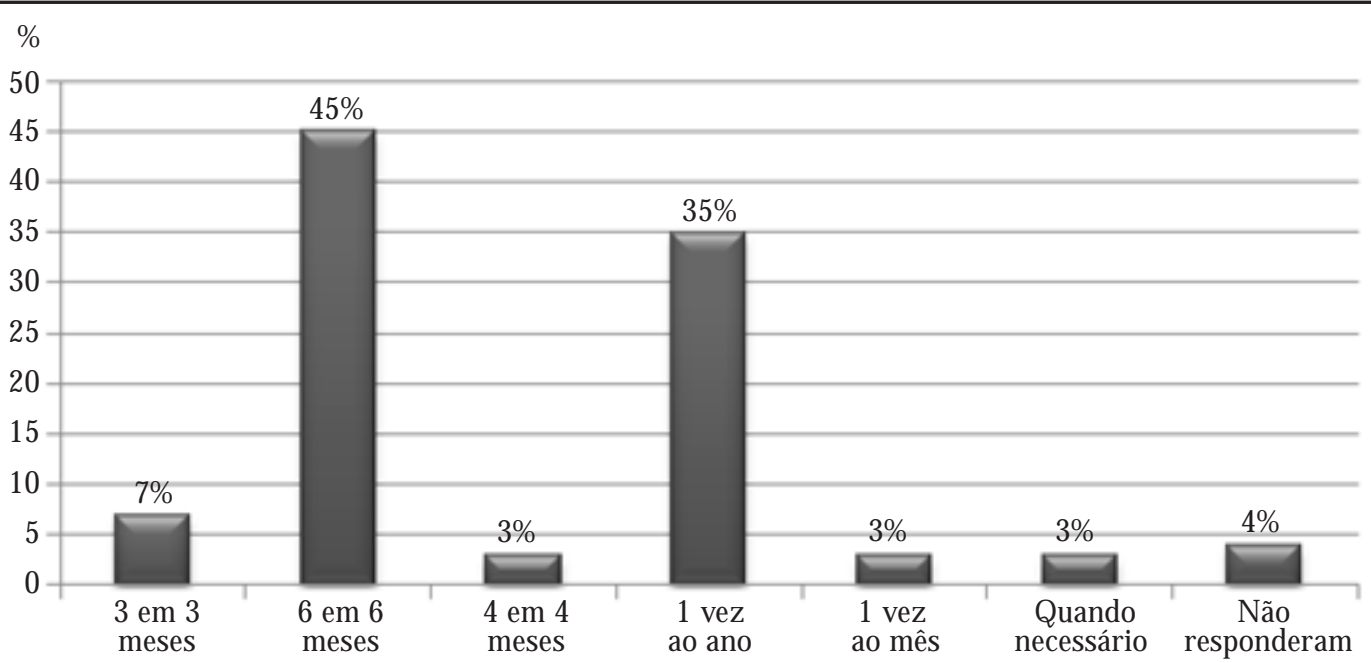

G ráfico 1 - Periodicidade do acompanhamento médico. Curitiba, PR, 2010.

Q uando questionados de como acham sua vida sexual depois dos 60 anos, 33,6\% (33) disseram que não perceberam grandes mudanças e 27,5\% (27) disseram ser melhor do que antes (G ráfico 2 ).

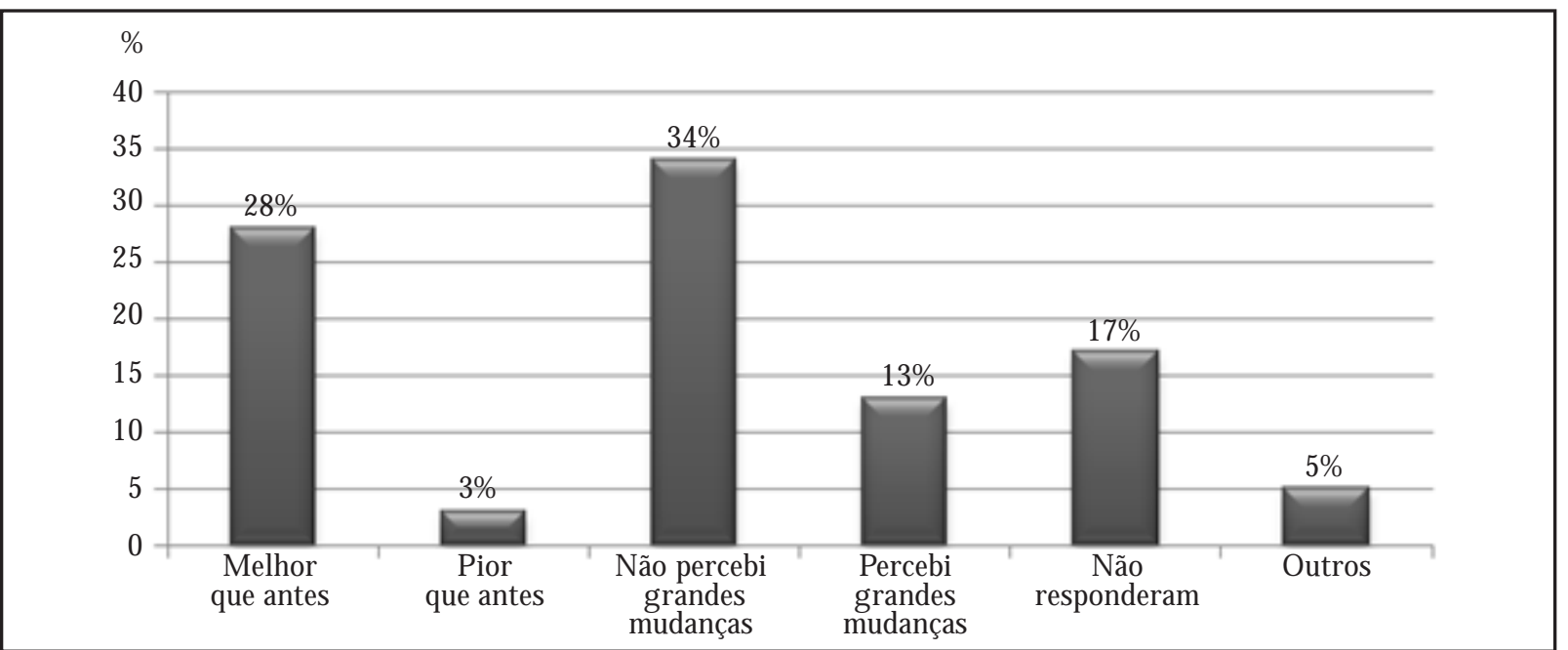

G ráfico 2 - Vida Sexual depois dos 60 anos. Curitiba, PR, 2010.

\section{DISCUSSÃO}

A AIDS nesse grupo etário traz à tona certos hábitos até então não revelados, como a sexualidade, escondida na pele enrugada e nos cabelos brancos, onde a libido é traduzida pelo preconceito ${ }^{(16)}$.

M uitos idosos abordados negaram-se a responder o questionário por vergonha, preconceito e por achar que este não é um assunto para se dis- 
cutir com "qualquer um". A pesar de muitos estudos mostrarem que o idoso ainda tem a sua sexualidade viva, ela é negada pela sociedade e por eles próprios. Isso é um fator cultural. São pessoas que vieram de uma época em que nem se cogitava falar sobre 0 assunto, quanto mais responder a um questionário sobre sua vida sexual para um desconhecido.

Quanto à predominância do sexo feminino entre 0 público entrevistado, diversas hipóteses explicam por que as mulheres vivem mais que os homens no Brasil. U ma delas diz respeito às diferenças na exposição a risco de acidentes de trabaIho, trânsito, homicídio e suicídio, a segunda hipótese está relacionada ao consumo de tabaco e álcool, favorecendo a ocorrência de doenças neoplásicas e cardiovasculares com maior frequência entre os homens; a última vincula-se à atitude em relação às doenças, em que as mulheres possuem maior adesão ao tratamento ${ }^{(17)}$.

0 grau de escolaridade dos entrevistados nos chamou atenção, somente $2 \%$ (2) não estudaram, ou seja, 98\% (96) podem buscar e conhecer informações sobre medidas de prevenção para DST e AIDS através de livros, revistas, inter net. Pessoas com grau de escolaridade mais avançado tendem a assimilar melhor as informações, e possuem maior facilidade de acesso aos serviços de saúde e de aquisição de preservativos ${ }^{(18)}$.

Quando avaliamos os dados em relação ao estado civil, observamos uma grande predominância de viúvos e divorciados. Estes dados indicam que após ficarem viúvos os idosos procuram por uma atividade para se "distrair" o que justifica os grupos dessa faixa etária serem bastante numerosos. Porém, os resultados demonstraram que dentre eles muitos não ter parceiro atualmente. Com parceiro fixo diminuem os riscos de exposição ao vírus HIV, tendo em vista que estudos epidemiológicos evidenciam que a multiplicidade de parceiros constitui um fator de risco para o contágio deDST eHIV/ AIDS ${ }^{(19)}$.

Em relação a ter atividade sexual nos últimos 12 meses, observa-se que a maioria teve relação e considera necessária a utilização de medidas de prevenção. Entendem a sua importância, visto que várias pessoas responderam que sabem que a AIDS não tem cura, que pode ser transmitida por via sexual e que é preciso se prevenir para ter uma boa saúde. Estes dados nos confirmam novamente que 0 idoso mantém a sua se- xualidade viva, diferente do que muitos possam pensar.

Porém, muitos idosos não se consideram vulneráveis à doença e a idéia de contrair HIV/ AIDS em uma idade avançada não existe, porque a informação sobre prevenção é direcionada quase exclusivamente aos jovens e a consciência sobre fatores de risco para idosos é baix ${ }^{(6,16)}$.

0 uso da camisinha, embora reconhecida pela maioria como meio de prevenção, não é frequentemente utilizada por esta população quando tem relações sexuais com pessoas de confiança(13). É necessário fazer com que a pessoa idosa perceba sua vulnerabilidade e esse é um dos desafios da prevenção. Porém, seu empoderamento passa pela superação de preconceitos. Os profissionais de saúde que atendem os idosos muitas vezes também não conseguem associar AIDS a pessoas idosas, pois a percepção do risco passa despercebida para essa população.

E m outro estudo realizado com idosos, os resultados demonstram que $98 \%$ dos entrevistados sabem que a relação sexual sem proteção pode transmitir a AIDS, cabendo ressaltar que 0 conhecimento da população sobre as formas de transmissão de doenças (neste caso, a AID S e DST ) não implica necessariamente na mudança de atitude, e como já citado anteriormente, o uso da camisinha não é ativo entre esta populaçãa (19).

Várias pesquisas realizadas com idosos demonstram que os mesmos associam a AIDS com a morte e a doença é percebida por eles como uma doença "ruim", "perigosa" sem cura, mas que a cultura do uso do preservativo não existe nessa população contribuindo, assim, para 0 aumento do número de casos da doença entre a terceira idade.

É um desafio diagnosticar pacientes soropositivos nessa faixa etária, por se tratar de mais um diagnóstico diferencial para um grupo já exposto a múltiplas patologias, o que leva a possibilidade de subnotificação de casos, ou se reflete em diagnósticos tardios e terapêuticas incorretas, acelerando a instalação de infecções oportunistas e de complicações ${ }^{(13)}$.

Com os avanços da tecnologia e da atenção à saúde, as pessoas da terceira idade vivem uma realidade nunca antes experimentada nesse período da vida. As drogas que atuam no desempenho sexual e as inovações na área da reposição hormonal aumentaram a qualidade e a frequência das relações sexuais ${ }^{(13)}$. 
L evando-se em consideração que a maioria da população estudada era do gênero feminino, consideramos que este acompanhamento foi devido ao fato delas irem ao ginecologista para realização do preventivo, e no caso dos homens, urologista para exame de próstata.

Os idosos podem relutar em falar com os médicos sobre sua vida sexual e os médicos podem relutar em fazer perguntas desse tipo. I sso resulta em negligenciar a possibilidade destas pessoas terem entrado em contato com o H IV ${ }^{(16)}$. U ma pesquisa realizada com idosos demonstra que a maioria absoluta dos casos de AIDS da pesquisa ocorreu por transmissão sexual, numa porcentagem de $96,6 \%{ }^{(6)}$.

Observa-se um bom nível de conhecimento a respeito da doença. Este fato pode estar ligado ao nível de escolaridade dos participantes, sendo que a maioria deles teve acesso, de alguma forma, à educação. U ma pesquisa realizada no Rio G rande do Sul, com idosos, mostra que dos 510 integrantes, 343 indicaram a afirmação que a AIDS tem cura, como falsa ${ }^{(20)}$.

Para as mulheres, o fato de terem relações sexuais desprotegidas é ainda mais preocupante, pois, fazer sexo sem camisinha é particularmente arriscado depois da menopausa, devido à fragilidade e ao ressecamento das paredes vaginais, às quais se tornam ainda mais finas, favorecendo 0 surgimento de ferimentos que abrem caminho para o HIV.

É importante lembrar que a realização de ações preventivas nas U nidades Básicas de Saúde, assim como a capacitação de seus profissionais, possibilitará que um maior número de pessoas idosas sejam orientadas sobre 0 assunto(15).

Aqui, entra novamente a questão da sexualidade. Sabendo-se que com o aumento da qualidade de vida e uma maior esperança de vida, as pessoas estão vivendo mais e melhor e assim, nas idades mais avançadas do viver, após os 60 anos, a população idosa continua sexualmente ativa. D esta forma, devemos nos preocupar com as doenças sexualmente transmissíveis na velhice, principalmente a AIDS ${ }^{(8)}$.

\section{CONSIDERAÇÕES FINAIS}

0 presente trabalho nos revela o quanto é relevante e necessário o desenvolvimento de programas de saúde pública que abordem este tema espe- cificamente para esta população, alertando para uma tomada de direcionamento quanto a propagação de informações preventivas frente às infecções de D ST 's e AIDS nesta população.

Observa-se que grande parte dos entrevistados ainda têm vida sexual ativa e poucos fazem uso de medidas de prevenção contra DST 's e AIDS, o que nos leva a repensar a necessidade de aprofundar a discussão sobre a vulnerabilidade a AIDS entre grupos de maior faixa etária. Porém, para uma abordagem mais integral da AIDS nessa população, há a necessidade, primeiro, de entender 0 processo biológico e cultural envolvido na sexualidade e o envel hecimento autônomo e saudável, no qual se vê atual mente a super ação da figura de um idoso dependente, doente e esperando sua morte.

Tornam-se necessárias estratégias educativas, realizadas por profissionais habilitados, para promover uma mudança no comportamento dos idosos, principalmente quanto às formas de prevenção.

A adoção de políticas de saúde pública que concentrem sua atenção na população mais velha, a realização de programas de prevenção voltada para 0 atendimento de pessoas com 60 anos ou mais deve estar atenta as questões de sexualidade no envelhecimento, onde será necessário quebrar tabus. A sexualidade nesta faixa etária não é discutida e, em alguns casos, é até ignorada. Os idosos devem ser vistos como indivíduos que possuem desejos, necessidades sexuais e fazem projetos para o futuro ${ }^{(6)}$.

\section{REFERÊ NCIAS}

1 Rodrigues RAP, Kusumota LM S, Fabrício SCC, Rosset-Cruz I, Lange C. Política $\mathrm{N}$ acional de Atenção ao I doso e a contribuição da enfer magem. Texto Contexto Enferm [ Internet] . 2007 [ citado 2011 jan $28]$;16(3):536-45. D isponível em: http:/ / www.scielo. $\mathrm{br} / \mathrm{pdf} / \mathrm{tcc} / \mathrm{v} 16 \mathrm{n} 3 / \mathrm{a} 21 \mathrm{v} 16 \mathrm{n} 3 . \mathrm{pdf}$.

2 M endes M RSSB, G usmão J L, F aro ACM , L eite RCBO. A situação social do idoso no Brasil: uma breve consideração. A cta Paul E nferm [ I nternet] . 2005 [ citado 2009 abr 30];18(4):422-6. Disponível em: http:/ / www.scielo.br/ pdf/ ape/ v18n4/ al1v18n4.pdf.

3 G arrido RM PR. 0 Brasil está envelhecendo: boas e más notícias por uma perspectiva epidemiológica. Rev Bras Psiquiatr [ I nternet] . 2002 [ citado 2009 abr 30] ;24(Supl 1):3-6. D isponível em: http:/ / www.scielo. $\mathrm{br} / \mathrm{pdf} / \mathrm{rbp} / \mathrm{v} 24 \mathrm{~s} 1 /$ 8849.pdf. 
4 Instituto Brasileiro de G eografia e Estatística. Perfil dos idosos responsáveis por domicílios no Brasil 1991 [ Internet] . Rio de Janeiro; 2002 [ citado 2009 maio 01] . Disponível em: http:/ / www.ibge.com.br/ home/ estatistica/ populacao/ perfilidoso/ tabela1_1.shtm.

5 Souza VC, Saldanha AAW, A raújo LF. Viver com AIDS na terceira idade. In: A nais do 70 Congresso Virtual HIV/AIDS; 2006 out 10; Lisboa, Portugal [ Internet] . Lisboa: SIDA net - A ssociação L usófona; 2006 [ citado 2010 jul 27]. Disponível em: http:/ / www.aidscongress.net/M odules/W ebC Aids Congress/ CommunicationH T M L .aspx?M id $=36 \&$ CommID $=324$

6 Pottes FA, Brito AM, Gouveia GC, A raújo EC, Carneiro RM . AIDS e envelhecimento: características dos casos com idade igual ou maior que 50 anos em Pernambuco, de 1990 a 2000. Rev Bras E pidemiol. 2007; 10(3):338-51.

7 M inistério da Saúde (BR), Programa Nacional de DST / AIDS. A epidemia de AID S no Brasil. Bol E pidemiol AIDST [ Internet]. 2009 [ citado 2010 jul 27];6(1). D isponível em: http:/ / www.aids.gov.br/ sites/ default/ files/ publicacao/ 2009/ boletim2009_ final_pdf_24513.pdf.

8 V ieira D LF C, Sobral B. 0 corpo envelhece, a sexualidade não: AIDS no diagnóstico diferencial entre as doenças comuns nos idosos. I nt Clin M ed [ Internet] . 2009 [ citado 2009 abr 01] ;2(B6). D isponível em: http:/ / www.medcenter.com/ medscape/ content. aspx?id $=18356 \&$ lang T ype $=1046$.

9 Gradim CVC, Sousa AM M , L obo JM. A prática sexual e o envelhecimento. Cogitare Enferm. 2007;12 (2):204-13.

10 A raújo VL B, Brito D M S, G imeniz M T, Queiroz TA, T avares CM . Características da Aids na terceira idade em um hospital de referência do Estado do Ceará, Brasil. Rev Bras E pidemiol [ I nternet] . 2007 [ citado 2010 abr 30] ;10(4):544-54. D isponível em: http:/ / ww w.scielosp.org/ pdf/ rbepid/ v10n4/ 12.pdf.

11 Sousa JL. Sexualidade na terceira idade: uma discussão da AIDS, envelhecimento e medicamentos para disfunção erétil. DST J Bras D oenças Sex Transm. 2008;20(1):59-64

\section{Endereço da autora / Dirección del autor / Author's address:}

L uciana P uchalski Kalinke

Curso de Enfermagem, Bloco Didático II,

Campus Botânico, Setor de Ciências da Saúde

Av. L othário M eissner, 632, Jardim Botânico

80210-170, Curitiba, PR

E-mail: lucianakalinke@yahoo.com.br
12 M inistério da Saúde (BR), Programa N acional de DST e AIDS. O controle da DST no Brasil [ I nternet] . Brasília (DF ); 2006 [ citado 2010 abr 30] . D isponível em: http:/ / bvsms.saude.gov.br/ bvs/ publicacoes/ manual_controle_das_dst.pdf.

13 Silva LS. Representações sociais de idosos sobre prevenção e transmissão da AIDS. A nais do 60 Congresso Virtual HIV/AIDS; 2005 out 15; Lisboa, Portugal [ I nter net] . L isboa: SIDA net - A ssociação L usófona; 2005 [ citado 2010 jul 24] . Disponível em: http:/ / w w w.aidscongress.net/ M odules/ W ebC A idsCongress/ Communication H T M L .aspx? M id= 35\& Comml D =181.

14 Dyniewicz AM. M etodologia da pesquisa em saúde para iniciantes. São Caetano do Sul: Difusão; 2007.

15 M inistério da Saúde (BR ), Conselho N acional de Saúde. Resolução 196, de 10 de outubro de 1996: diretrizes e normas regulamentadoras de pesquisa envolvendo seres humanos. Brasília (D F ); 1996.

16 Leite M T, M oura C, Berlezi E M. Doenças sexualmente transmissíveis e HIV/ AIDS na opinião de idosos que participam de grupos de terceira idade. Rev Bras G eriatr Gerontol. 2007;10(3):339-54.

17 Silva RM. Perfil dos idosos frente a prevenção do vírus HIV no município de M uriaé/ M G : a importância da assistência de enfermagem [ monografia] . M uriaé: Curso de Bacharelado em E nfer magem, F aculdade de M inas; 2008 [ citado 2010 jul 24] . D isponível em: http:/ / www.artigosonline.com.br/ 1185/ perfil-dos-idosos-frente-a-prevencao-do-virushiv-no-municipio-de-muriaemg-importancia-daassistencia-de-enfermagem.

18 Sá AM S, Callegari F M , Pereira ET. Conviver com HIV/Aids: concepções de pessoas com idade acima de 50 anos. Ser Soc. 2007;(21):259-84.

19 Lima M M . Conhecimento da população de Viçosa, M G, sobre as formas de transmissão da AIDS. Ciênc Saúde Colet. 2008;13(6):1879-88.

20 L azzarotto AR. O conhecimento de HIV/AIDS na terceir a idade: estudo epidemiológico no Vale dos Sinos. Ciênc Saúde Colet. 2008;13(6):1833-40.

Recebido em: 13/ 02/2011

A provado em: 22/ 07/ 2011 